\title{
Healthcare Costs of Inflammatory Myopathies
}

\author{
SASHA BERNATSKY, PANTELIS PANOPALIS, CHRISTIAN A. PINEAU, MARIE HUDSON, YVAN ST. PIERRE, \\ and ANN E. CLARKE
}

ABSTRACT. Objective. Little information exists regarding the economic burden related to inflammatory myopathies. Our objective was to estimate health services costs in a large, unselected, population-based sample of patients with inflammatory myopathies.

Methods. We identified subjects with polymyositis and dermatomyositis from administrative healthcare databases (covering all beneficiaries, about 7.5 million) in Quebec province, Canada. Average estimates of health services costs (physician visits, diagnostic tests and procedures, outpatient surgeries and procedures, acute care hospitalizations) for 2003 were calculated by multiplying health service use levels by the appropriate unit prices, determined from government fee schedules and other sources. Multiple linear regression analyses were performed to establish whether specific factors (age, sex, disease duration, region of residence, socioeconomic status, type of myositis, disease severity) were associated with cost.

Results. We identified 1102 subjects with inflammatory myopathy from January 1, 1989, to January 1, 2003. About two-thirds were women (68.9\%); average age at case ascertainment was 57.4 years (SD 18.4). The average cost of all reimbursed health services, in 2008 Canadian dollars, was \$4099 per patient (SD \$9639). Costs increased with age, and were highest early in the disease course. Greater disease severity (defined as the need for prior hospitalization for myositis) was also a strong predictor of both physician costs and total costs.

Conclusion. These results indicate significant economic burden related to inflammatory myopathies, with important demographic predictors. Our estimates suggest that the health services costs in inflammatory myopathies may equal, or exceed, those of other serious diseases, such as rheumatoid arthritis and systemic sclerosis. (First Release March 1 2011; J Rheumatol 2011; 38:885-8; doi:10.3899/jrheum.101083)

Key Indexing Terms:

DERMATOMYOSITIS

HEALTH SERVICES RESEARCH

\section{EPIDEMIOLOGY \\ POLYMYOSITIS}

Autoimmune myopathies are potentially debilitating (or even life-threatening) diseases ${ }^{1}$ characterized by muscle inflammation (myositis) with subsequent weakness. Two classic forms are polymyositis (PM) and dermatomyositis (DM). Since persons with inflammatory myopathies are at

From the Divisions of Clinical Epidemiology, Rheumatology, and Allergy and Clinical Immunology, McGill University Health Centre (MUHC); and Division of Rheumatology, Jewish General Hospital, Montreal, Quebec, Canada.

Dr. Bernatsky is the recipient of career awards from the Canadian Institutes for Health Research (CIHR), Fonds de recherche en santé du Québec (FRSQ), and the Canadian Arthritis Network (CAN), and of support from the MUHC Department of Medicine and Research Institute. Dr. Hudson is the recipient of career awards from CIHR and CAN. Dr. Pineau is supported by MUHC Research Institute and Department of Medicine. Dr. Clarke is an FRSQ national scholar.

S. Bernatsky, MD, PhD, MUHC, Divisions of Clinical Epidemiology and Rheumatology; P. Panopalis, MD, MSc; C.A. Pineau, MD, MUHC, Division of Rheumatology; M. Hudson, MD, MSc, Division of Rheumatology, Jewish General Hospital; Y. St. Pierre, MSc, MUHC, Division of Clinical Epidemiology; A.E. Clarke, MD, MSc, MUHC, Divisions of Clinical Epidemiology and Allergy and Clinical Immunology.

Address correspondence to Dr. S. Bernatsky, Division of Clinical Epidemiology, Research Institute of the McGill University Health Centre, 687 Pine Avenue West, V-Building, Montreal, Quebec H3A 1A1, Canada. E-mail: sasha.bernatsky@mail.mcgill.ca

Accepted for publication December 29, 2010. risk for substantial morbidity and disability ${ }^{2,3}$, these diseases, although relatively uncommon, are believed to have an important effect on healthcare costs. The most effective treatments for these diseases include corticosteroids, steroid-sparing agents, and intravenous immunoglobulin.

We previously evaluated the prevalence of inflammatory myositis in Quebec, demonstrating PM and DM to affect as many as 1 person in $5000^{4}$. However, estimates of the economic burden of inflammatory myopathies have not been published, to our knowledge. Our objective thus was to estimate health services costs in a large sample of patients with inflammatory myopathies, and to evaluate potential predictors of cost in this population.

\section{MATERIALS AND METHODS}

In Canada, each province's healthcare system is publicly funded and mandated to be universal and comprehensive. We based our analyses on a cohort of patients assembled using physician billing and hospitalization databases from the Canadian province of Quebec. We identified diagnoses of inflammatory myositis from January 1, 1989, to January 1, 2003.

The billing database documents physician services (visits and procedures) for all Quebec citizens seeking provincially reimbursed healthcare; the hospital database records all hospitalizations, including discharge diagnoses and length of stay. For both databases, diagnoses are provided as International Classification of Diseases codes (e.g., DM 710.3; PM 710.4).

Personal non-commercial use only. The Journal of Rheumatology Copyright @ 2011. All rights reserved. 
Cases in our sample were defined by either of the following: (1) two or more relevant physician billing diagnoses, 2 months apart but within a 2year span; or (2) any hospitalization with a relevant (primary or nonprimary) discharge diagnosis. A similar approach to identifying cases of inflammatory myopathies from administrative data has been shown to have $88.4 \%$ sensitivity and $97.1 \%$ specificity, compared to rheumatology records 5 .

For identified patients who remained alive as of January 1, 2003, we determined health services costs over the following year. This included all physician service use (visits, outpatient procedures, and surgeries), major diagnostic tests (primarily imaging) and diagnostic procedures (e.g., biopsy), and acute-care hospitalizations. Average costs of health services were calculated by multiplying health service use levels by the appropriate unit prices. For physician visits, procedures, and diagnostic tests, costs were determined from government fee schedules ${ }^{6}$. Acute-care hospital costs were based on data from the Ontario Case-Costing Initiative, adjusted to reflect Quebec healthcare wages ${ }^{7}$. We generated an estimate of these health services costs, in 2008 Canadian dollars, using inflation data based on the Bank of Canada's consumer price information ${ }^{8}$. As has traditionally been done for rheumatic diseases ${ }^{9}$, estimates include all services received, regardless of whether they were provided for myositis or a comorbid condition.

Multiple linear regression analyses were performed to establish whether specific factors were associated with health services costs in the myositis sample. Covariates included available demographics (age, sex, region of residence, and local income as a marker for socioeconomic status) and disease duration. These variables were included because they have been shown as potential factors of importance, influencing medical costs in other rheumatic diseases ${ }^{10,11,12}$. Our income covariate was defined using postal codes and information from the Statistics Canada Census on each subject's neighborhood income level (grouped into tertiles). Disease duration was based on the first date that each patient had a physician visit or hospitalization coding for myositis, within the administrative databases. Because disease severity also has been noted to play a potential role in medical costs for patients with rheumatic diseases ${ }^{13,14}$, we also assessed the effect (on cost) of whether a patient had ever required hospitalization for the inflammatory myopathy (based on primary diagnoses in the hospitalization database). A variable for disease type (PM vs DM) was also included in the model.

The distribution of healthcare costs, in dollars, deviated from normal. Thus, for the regression models, we transformed the outcome by taking the natural logarithm of cost, which has a more normal distribution. Hence, the coefficients for the variables in the model can be thought of as approximate percentage change in cost, rather than additional dollars due to a unit change in a predictor.

\section{RESULTS}

We identified 1102 cases of inflammatory myopathies in the provincial database. The majority of the subjects were women (68.9\%). The average age of the subjects was 57.4 years (SD 18.4); the average disease duration as of 2003 was 6.3 years (SD 4.0). Regarding economic burden, the average cost of all reimbursed health services, in 2008 Canadian dollars, was $\$ 4099$ per patient (SD \$9639). This reflects the total cost of all reimbursed health services, per patient, including the cost of comorbidity; the large SD indicates the high variability of health costs among individuals. Hospital costs contributed a high proportion $(74 \%)$ of health services costs, with physician visits contributing most of the remaining costs. About a quarter (24.2\%) of patients studied had a hospitalization in 2003.

Table 1 provides a summary of potential determinants of cost, on the logarithmic scale. Age, as well as being a significant predictor of total costs, was associated specifically with physician services costs, and was the main predictor of hospital costs. There was also evidence of decreasing total costs with increasing disease duration, which may have been due to a declining requirement for physician services over time. Being a woman was a predictor of costs related to physician services, but not hospitalization. Our marker of disease severity (requirement for hospitalization for myositis prior to 2003) was a significant predictor of both total costs and hospital costs.

\section{DISCUSSION}

Our analyses used administrative data, a situation that precludes clinical confirmation of our cases; however, using our "composite" case definition based on either 2 or more physician visits or 1 or more hospitalizations, the prevalence estimate of inflammatory myositis in Quebec for 2003 is estimated at 15.6 cases per $100,000^{4}$. Accounting for incomplete case ascertainment, the prevalence in our aging population could be as high as 21.5 per 100,000 . We relied on the International Classification of Diseases, 9th ed., which has specific codes for PM and DM, but does not have a specific code for a related condition, inclusion body myositis (IBM). Since IBM is often initially clinically difficult to distinguish from pure inflammatory myopathies, it is likely that some of the PM cases we identified through our administrative datasets were actually IBM. However, stricter, clinical criteria for case identification may miss some cases of autoimmune myopathy, as noted ${ }^{15}$. Thus, our approach, combining both hospitalization and billing data, seems to be a reasonable attempt to maximize sensitivity and specificity.

We have confirmed that the economic burden associated with inflammatory myopathies is substantial. In our multivariate regression analyses, we found evidence that age, shorter disease duration, and our marker of disease severity, were all independently associated with higher health services costs.

Age is a fairly consistent correlate of medical costs, and in part may reflect the greater accumulation of comorbidity (such as arterial events) that occurs with age ${ }^{3}$. As well, in our sample, age (treated continuously) was itself a predictor for the outcome of hospitalization in a separate logistic regression (the OR for hospitalization, due to age, was 1.02, 95\% CI 1.01, 1.03). Thus, much of the effect of age as a driving force behind cost is related to hospitalizations (although age was also clearly associated with higher physician costs and higher total health services costs).

That being a woman was a predictor of physician costs may reflect a greater likelihood for women to seek medical care rather than a tendency for greater disease severity in women compared to men. In fact, it has been noted that women with DM are less likely to present with florid muscle weakness ${ }^{16}$ and more likely to have a favorable course, 
Table 1. Multivariate regression: predictors of health services costs in inflammatory myopathies $(\mathrm{n}=1102)$ in 2003. The outcome was the natural logarithm of health services costs, in 2008 Canadian dollars.

\begin{tabular}{lccc}
\hline Predictor & \multicolumn{3}{c}{ Regression Coefficients } \\
& $\begin{array}{c}\text { Physician } \\
\text { Services }\end{array}$ & $\begin{array}{c}\text { Acute Care } \\
\text { Hospital Costs }\end{array}$ & $\begin{array}{c}\text { Total } \\
\text { Costs* }\end{array}$ \\
\hline Being a woman & $0.42(0.20,0.65)$ & $-0.05(-0.53,0.43)$ & $0.39(0.11,0.67)$ \\
Age & $0.02(0.01,0.02)$ & $0.04(0.02,0.05)$ & $0.03(0.02,0.03)$ \\
Disease duration & $-0.04(-0.07,-0.01)$ & $-0.05(-0.11,0.01)$ & $-0.05(-0.08,-0.02)$ \\
Prior hospitalization for myositis & $0.17(-0.05,0.39)$ & $0.89(0.42,1.37)$ & $0.34(0.07,0.61)$ \\
Polymyositis (vs dermatomyositis) & $0.13(-0.09,0.35)$ & $0.29(-0.19,0.77)$ & $0.18(-0.09,0.45)$ \\
Residence outside Montreal & $-0.19(-0.40,0.03)$ & $0.16(-0.31,0.63)$ & $-0.12(-0.38,0.15)$ \\
Upper tertile income area** & $-0.37(-0.69,-0.05)$ & $-0.91(-1.61,-0.22)$ & $-0.52(-0.92,-0.13)$ \\
\hline
\end{tabular}

Adjusted R values are 0.063 for physician services, 0.043 for acute care hospital costs, and 0.067 for total costs

* Includes physician services, hospital costs, diagnostic tests and procedures, and outpatient surgeries and procedures. ** The reference group for income is the middle and lower tertiles combined.

as compared to men. Our results suggested decreased costs in patients with an income in the upper-tertile income bracket, which again suggests the importance of socioeconomic status as a predictor of treatment outcome.

We found that the highest costs for inflammatory myopathies were incurred early in the disease course. In contrast, in at least 1 study, the costs related to patients with primary Sjögren's syndrome increased over time, while there was no appreciable change for a comparator group with rheumatoid arthritis (RA) ${ }^{11}$. Because inflammatory myopathies have been associated with underlying malignancies, some of the cost early in the disease course may be due to the fairly extensive laboratory investigations that are done to rule out this complication (especially imaging), but we note that diagnostic imaging was not, on average, a particularly high-cost component of health service use. However, some of the cases were diagnosed over 20 years ago, and current costs may in fact be higher, for example because of the increasing availability of imaging and other expensive cancer screening tests.

One potential message to be taken from our work is that hospital costs are an important component of the economic burden generated by inflammatory myopathies. For reasons both economic and social, this may indicate the importance of prompt referral, diagnosis, and treatment (which might prevent the need for hospitalizations), especially since delay to diagnosis has been shown to be a predictor of poor outcome in $\mathrm{DM}^{2}$. Of course, our study does not provide specific evidence to support this hypothesis. It may be that higher hospital costs are due to profound impairment in mobility for patients with inflammatory myopathy who have a very florid and catastrophic presentation requiring hospitalization. In that event, the recognition of high costs related to hospitalization can still encourage prompt treatment that might allow earlier discharge, for example.

Table 2 compares our findings to cost estimates for a similar array of healthcare services in other serious rheumatic diseases, such as $\mathrm{RA}^{9}$ and systemic sclerosis ${ }^{17}$. The bottom
Table 2. Comparisons of average annual health service cost estimates (Canadian dollars, 2008, per patient) from studies for rheumatoid arthritis, systemic sclerosis, and inflammatory myopathies.

\begin{tabular}{lccc}
\hline $\begin{array}{l}\text { Types of } \\
\text { Expenses }\end{array}$ & $\begin{array}{c}\text { Rheumatoid } \\
\text { Arthritis* }\end{array}$ & $\begin{array}{c}\text { Systemic } \\
\text { Sclerosis** }\end{array}$ & $\begin{array}{c}\text { Inflammatory } \\
\text { Myopathies }\end{array}$ \\
\hline a. Healthcare visits & 463 & 766 & 630 \\
b. Diagnostics & 580 & 760 & 238 \\
c. Outpatient surgeries & 305 & 229 & 201 \\
d. Hospitalizations & 2047 & 1494 & 3030 \\
Items a + b + c & 1348 & 1755 & 1069 \\
All $(\mathrm{a}+\mathrm{b}+\mathrm{c}+\mathrm{d})$ & 3395 & 3249 & 4099 \\
\hline
\end{tabular}

* Based on health resource use data from 4351 patients $^{9}$. ** Based on annual questionnaires in 457 patients. Includes nonphysician healthcare vistis $^{17 .}$

row of the table provides average annual cost estimates for health services for each disease in 2008 Canadian dollars. This suggests that the mean financial burden due to inflammatory myopathies may be at least as high, or higher, compared to other complex rheumatic diseases, such as RA and systemic sclerosis.

To our knowledge, no other detailed studies of the economic burden of inflammatory myopathies have been published to date. Our study is novel, but our dependence on administrative databases does entail important restrictions on the type of cost elements that we could study. We did not assess indirect costs (that is, the economic burden related to lost work productivity), but given the high disability in patients with inflammatory myopathies, indirect costs are likely to be considerable as well ${ }^{2}$. Regarding our evaluation of medical services costs, we were able to use administrative data to assess the major contributors to direct costs, except for medications (which are not comprehensively recorded in Quebec's prescriptions database) and assistive devices.

Our estimate of health services costs does not include allied care (e.g., physical and occupational therapy, etc., which are not recorded in Quebec's administrative data) and our diagnostic test category does not include high-volume 
but relatively low-cost routine laboratory testing (e.g., hematology and chemistry, not recorded in the administrative data). Hence, our estimates of health services costs in inflammatory myopathies are likely fairly conservative, compared to other studies in rheumatic diseases such as RA. We are planning to do further work on direct costs, looking at drugs in a subset of patients for whom administrative data are available.

Our work emphasizes the high cost of inflammatory myopathies. For patients with inflammatory myopathies, health services costs seem to be at least as great as, or greater than, those for patients with other serious rheumatic diseases, such as RA and systemic sclerosis. The estimates we produced will be useful to researchers, consumers, decision makers, and others who are interested in the economic burden of inflammatory myopathies.

\section{REFERENCES}

1. Airio A, Kautiainen H, Hakala M. Prognosis and mortality of polymyositis and dermatomyositis patients. Clin Rheumatol 2006;25:234-9.

2. Rider LG, Lachenbruch PA, Monroe JB, Ravelli A, Cabalar I, Feldman BM, et al; IMACS Group. Damage extent and predictors in adult and juvenile dermatomyositis and polymyositis as determined with the myositis damage index. Arthritis Rheum 2009;60:3425-35.

3. Tisseverasinghe A, Bernatsky S, Pineau CA. Arterial events in persons with dermatomyositis and polymyositis. J Rheumatol 2009;36:1943-6.

4. Bernatsky S, Joseph L, Pineau CA, Bélisle P, Boivin JF, Banerjee $\mathrm{D}$, et al. Estimating the prevalence of polymyositis and dermatomyositis from administrative data: age, sex and regional differences. Ann Rheum Dis 2009;68:1192-6.

5. Bernatsky S, Linenham T, Hanly JG. The accuracy of administrative data diagnoses of systemic autoimmune rheumatic diseases. J Rheumatol 2011 [in press].

6. La Régie de l'assurance maladie du Québec Tarif des actes médicaux, Quebec. Quebec City: RAMQ; 2003. [Internet. Accessed February 14, 2011.] Available from: http://www.ramq.gouv.qc.ca/fr/ professionnels/medspe/manuel/manu_tdm.shtml
7. Ontario guide to case-costing. Ontario Case-Costing Initiative, Ministry of Health and Long-Term Care, Health Data Branch, Data Standards Unit, Toronto, 2010. [Internet. Accessed January 13, 2011.] Available from: http://www.occp.com

8. Bank of Canada. Rates and statistics inflation calculator. [Internet. Accessed January 13, 2011.] Available from: http://www.bankofcanada.ca/en/rates/inflation_calc.html

9. Huscher D, Merkesdal S, Thiele K, Zeidler H, Schneider M, Zink A, for the German Collaborative Arthritis Centres. Cost of illness in rheumatoid arthritis, ankylosing spondylitis, psoriatic arthritis and systemic lupus erythematosus in Germany. Ann Rheum Dis 2006;65:1175-83.

10. Clarke AE, Zowall H, Levinton C, Assimakopoulos H, Sibley JT, Haga M, et al. Direct and indirect medical services costs incurred by Canadian patients with rheumatoid arthritis: a 12 year study. J Rheumatol 1997;24:1051-60.

11. Callaghan R, Prabu A, Allan RB, Clarke AE, Sutcliffe N, St. Pierre $\mathrm{Y}$, et al. Direct healthcare costs and predictors of costs in patients with primary Sjogren's syndrome. Rheumatology 2007;46:105-11.

12. Bernatsky S, Dobkin PL, De Civita M, Penrod JR. Co-morbidity and physician use in fibromyalgia. Swiss Med Wkly 2005; 135:76-81.

13. Lacaille D, Clarke AE, Bloch DA, Danoff D, Esdaile JM. The impact of disease activity, treatment and disease severity on short-term costs of systemic lupus erythematosus. J Rheumatol 1994;21:448-53.

14. Bernatsky S, Duffy C, Malleson P, Feldman DE, St. Pierre Y, Clarke AE. Economic impact of juvenile idiopathic arthritis. Arthritis Rheum 2007;57:44-8.

15. Troyanov Y, Targoff IN, Tremblay JL, Goulet JR, Raymond Y, Senecal JL. Novel classification of idiopathic inflammatory myopathies based on overlap syndrome features and autoantibodies: analysis of 100 French Canadian patients. Medicine 2005;84:231-49.

16. Klein R, Teal V, Taylor L, Troxel A, Werth V. Number, characteristics, and classification of patients with dermatomyositis seen by dermatology and rheumatology departments at a large tertiary medical center. J Am Acad Dermatol 2007;57:937-43.

17. Bernatsky S, Hudson M, Panopalis P, Clarke AE, Pope J, Leclercq $\mathrm{S}$, et al; Canadian Scleroderma Research Group. The cost of systemic sclerosis. Arthritis Rheum 2009;61:119-23. 